\title{
GPU4S: Embedded GPUs in Space
}

\author{
Leonidas Kosmidis*, Jérôme Lachaize ${ }^{\dagger}$, Jaume Abella* \\ Olivier Notebaert $^{\dagger}, \quad$ Francisco J. Cazorla*, ${ }^{*}$, David Steenari ${ }^{\S}$ \\ * Barcelona Supercomputing Center (BSC), Spain \\ ${ }^{\dagger}$ Airbus Defence and Space, France \\ ${ }^{\ddagger}$ Spanish National Research Council (IIIA-CSIC), Spain \\ ${ }^{\S}$ European Space Agency, The Netherlands
}

\begin{abstract}
Following the same trend of automotive and avionics, the space domain is witnessing an increase in the on-board computing performance demands. This raise in performance needs comes from both control and payload parts of the spacecraft and calls for advanced electronics able to provide high computational power under the constraints of the harsh space environment. On the non-technical side, for strategic reasons it is mandatory to get European independence on the used computing technology. In this project, which is still in its early phases, we study the applicability of embedded GPUs in space, which have shown a dramatic improvement of their performance per-watt ratio coming from their proliferation in consumer markets based on competitive European technology. To that end, we perform an analysis of the existing space application domains to identify which software domains can benefit from their use. Moreover, we survey the embedded GPU domain in order to assess whether embedded GPUs can provide the required computational power and identify the challenges which need to be addressed for their adoption in space. In this paper, we describe the steps to be followed in the project, as well as the results of our preliminary analyses in the first months of the project.
\end{abstract}

\section{INTRODUCTION AND BACKGROUND}

The space market is in constant search for high performance, scalable processing solutions to satisfy the increased computational needs of future missions for increased autonomy and data processing. While reusing solutions from other domains can reduce non-recurrent costs, space has its unique set of constraints. Increased performance demands are required for the platform computers, which are in charge of controlling critical functionalities like the spacecraft power distribution, navigation and guidance, as well as for the payload computers (in charge of controlling the payload devices, and preprocessing acquired payload data before its transmission to the ground) to process more data.

Graphics processing units (GPUs), initially a special purpose type of accelerator for visualisation tasks, have since several years ago outperformed Central Processing Units (CPUs) raw performance and energy efficiency. This opens the door to achieve unprecedented performance with a very high energy efficiency for demanding computations, becoming essential for high-performance computing. As a matter of fact, one quarter (125) of the supercomputers in the recent edition of the TOP500 / Green500 list (as of June 2019) are based on GPUs, including the two most powerful supercomputers. Past studies analysed the applicability of high-performance GPUs in space [1][2]. Those studies concluded that although their energy efficiency is high, their power consumption is an order of magnitude higher than the limited power budget of a space system, which is limited to a couple of Watts.

Interestingly, GPUs entered in the embedded domain to satisfy the increasing demand for multimedia-based handheld and consumer devices such as smartphones, in-vehicle entertainment systems, televisions, set-top boxes etc. They were re-designed compared to their high-performance desktop/server counterparts to exhibit low-power requirements, essential for battery power and thermally-constrained devices. Improvements in the transistor technology allowed achieving impressive performance capabilities that were only possible in high performance systems of the past decade [3]. Although the GPU vs CPU performance ratio is lower in the embedded domain than in the high-performance one due to power and thermal constraints, mobile GPUs are increasingly considered for accelerating heavy workloads, for applications ranging from signal processing, to advanced driving assistance systems (ADAS) in cars, as well as prototype supercomputers for exascale [4][5].

Despite their promising characteristics, embedded GPUs have not been explored for their applicability in this domain. This project aims at covering this gap by providing an initial assessment of existing embedded GPUs, as a first step of their further exploration and adoption in this domain.

The rest of the paper is organised as follows. Section II introduces the project, which includes the on-going and future activities. Section III analyses the performance demand of current and future space missions. Section IV details the outcome of work on the analysis of the embedded GPU market. Finally, Section V describes the future work and Section VI presents the main conclusions of our work so far.

\section{THE PROJECT}

The project explores the suitability of embedded GPUs for space from both, software and hardware perspectives. In order to reach its main goal, the project is organised in 4 main activities as shown in Figure 1.

Space mission analysis: In this activity, we survey potential space areas and particular algorithms and applications that can benefit from the use of GPUs in space, ensuring that the identified applications are suitable for the GPU execution 


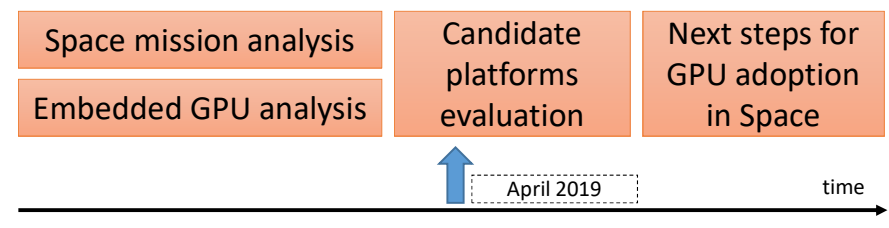

Fig. 1. Main activities. Box width does not represent activity duration

model. This is important because there are fundamental design differences between GPUs and CPUs which are required to be taken into account to understand how certain algorithms used in space, e.g. those with divergent path execution among different threads or those not performing coalesced memory accesses, behave differently in embedded GPUs w.r.t. highperformance GPUs. In particular, certain space algorithms may not be suitable for embedded GPUs despite their high computational nature, and might require to be redesigned.

We provide a preliminary list of applications already validated as GPU-compatible in Section III. Moreover, we define a preliminary set of criteria that can be used for the selection of a GPU candidates depending on the mission profile.

Embedded GPU analysis: In parallel to previous activity, we survey the available commercial-of-the-shelf (COTS) hardware and soft-IP (Intellectual Property), in order to identify their characteristics with the goal of selecting a candidate board for evaluation in the next activities. We mainly focus on European IP, which in turn dominates the market and can provide complete independence in the European space sector. In this line, in order to understand the wide range of embedded GPUs, we perform a taxonomy of existing products.

In addition to the mission profile criteria from the Space mission analysis, an important aspect for the selection of a candidate board, is the performance and energy-efficiency characteristics, due to the fundamental architectural differences between their designs and that of their high-performance counterparts, which need to be exploited by the software. This fact has only been superficially explored up to date [6], with many works in the literature overlooking these differences and treating them as equivalent. Another equally important aspect is the available software ecosystem that is crucial for the applicability in space. Moreover, we identify software applications in the space domain that can benefit from GPUs and justify their use. Furthermore, it is important to ensure that these applications and their corresponding algorithms fit the programming model of GPUs.

Next, we survey the potential market options in order to select the appropriate candidates for evaluation. We started by providing a taxonomy of the available GPU options, listing the characteristics and the differences between products from different vendors or families of GPUs in each category, namely GPUs and soft-IP.

The classification and the summary of our preliminary observations of the survey are presented in Section IV.

Candidate Platform Comparison: From both previous activities, we will identify promising GPU candidate plat- forms. As a next step we will perform a thorough comparison between the selected embedded GPU candidates and existing and future processing devices for the space domain. This way, the potential benefits of embedded GPUs can be evaluated, specifically regarding the high-performance requirements of future missions, while respecting the power and thermal limitations of the space environment. In order to do this, we will perform the evaluation by porting representative kernels derived from existing space algorithms to the GPU. Moreover, performance and other data from past missions and previous Application-Specific Integrated Circuit (ASIC) processes will be used for comparison, by normalising them according to the current space technology node $(65 \mathrm{~nm})$. This will allow the selection of the most appropriate GPU platform for space from the evaluated candidates.

Next Steps for GPU adoption: As final step of the project, we will define the future steps required towards the adoption of GPUs in space, by identifying current limitations and proposing appropriate solutions to overcome them. In order to assess the next steps for the adoption of GPUs in the space domain with a system integrator view, we will examine the necessary steps for qualification of COTS systems by addressing their reliability concerns at system level or the development of radiation hardened components. In the former case we will also provide software fault-tolerance solutions specifically designed for these platforms, in case a COTS embedded GPU is selected in the previous task. Finally, an ESA provided algorithm will be ported to the GPU platform.

\section{Space Application Survey}

In this Section, we examine the space applications domain regarding the use of embedded GPUs. The performance requirements of space missions are constantly increasing. As an example of current missions, the Gaia astrometry mission (devoted to the measurement of the positions of celestial bodies), launched in 2013 by the European Space Agency as a follow-up mission to the Hipparcos mission in 1989, has a hundred times better accuracy and aims to map 1.7 billion stars, 4 orders of magnitude more stars than its predecessor. Additionally, scientific missions can generate a massive amount of data, which may be challenging to transmit even on earth's surface. Most on-board scientific instruments, including the ones used for observation, use arrays of sensors, which allow for parallel processing. For example, the Alpha Magnetic Spectrometer (AMS-02) instrument at the International Space Station (ISS) [7], produces data in the rate of 7 Gigabit/s. For this reason, it uses an array of 600 CPUs to reduce the amount of data by 3 orders of magnitude, before transmission [8].

Finally, future space missions such as the Active Debris Removal (ADR) will need an unparallelled amount of computational power to achieve new functionalities, for autonomous guidance and navigation control (GNC) based on image processing and machine learning [9], in order to identify, approach, rendez-vous and finally remove the target. Similarly, the on board data processing for next-generation missions will increase in almost every kind of mission [10][11][12]: 
- For Science and robotic exploration, high data performance acquisition chains will be required to meet the high data rate generated by instruments (spectrometers, imagers etc.) as the importance of on board-autonomy and processing needs for planetary exploration is increased.

- For Earth Observation, the evolution in sensor resolution, dynamic range and faster readout rates has led to a dramatic increase of sensor bandwidth and data volume, creating significant bottlenecks in downlink capacity and a need of very high on-board data processing capabilities for both data processing and compression. Moreover, new technologies such as deforming mirrors, require important on-board processing. Video from space could also be a new application, as well as the image enhancement based on the acquisition of several images.

- For next generation launchers, considerably increasing data rate and on-board processing capabilities can enable interesting applications, such as increased user telemetry transmission channels. This includes the transmission of video data to monitor critical agile launch manoeuvres, which are not possible with todays transmission rates of up to $400 \mathrm{Mbits} / \mathrm{s}$ with a compression ratio of 20 .

- The navigation equipment for new missions like the space tug concept required for Active Debris Removal (ADR) [9] which will be able to change the orbit of non-cooperative spacecraft, will be based on computer vision. This type of processing, including localisation and mapping, is essential and very demanding.

- In the telecommunication domain, future applications go beyond the classical geosynchronous equatorial orbit (GEO) based telecommunication systems, and include missions such as spectrum surveillance or machine to machine communication from the Low Earth Orbit with medium or large spacecraft constellations.

- Radar processing is also expected to follow a technological evolution similar to and synergistic with the telecommunication domain, since both require high-performance signal processing.

- Launchers is the only domain in which performance requirements are not expected to grow. For expandable launchers, requirements for high performance processing are not growing very fast since the need for video transmission during the flight (mainly for post-mortem analysis) requires a strong data reduction ratio: in order of 20. This is currently covered and no fast evolution of the need is foreseen, remaining in a similar range to the current Earth Observation requirement. On reusable launchers, the re-entry/landing phases will require high performance highly available processing devices especially for vision based navigation and guidance for landing. In this study, such application is considered as similar with the Planetary Approach and Landing already covered by the exploration domain. So the launcher use case will not be particularly considered further in this study.

- Finally, future missions will require significant autonomy and agility provided by advanced robotics for in-orbit
TABLE I

EXAMPLE SPACE DOMAINS AND REPRESENTATIVE ALGORITHMS, SUITABLE FOR GPU ACCELERATION.

\begin{tabular}{|l|l|l|}
\hline \multicolumn{1}{|c|}{ Use Case type } & \multicolumn{1}{|c|}{ Algorithm name } & \multicolumn{1}{c|}{ Algorithm description } \\
\hline $\begin{array}{l}\text { Science/Image } \\
\text { processing }\end{array}$ & GAIA VPU & $\begin{array}{l}\text { Complex Image Processing } \\
\text { chain (49 algorithms } \\
\text { pipelined) }\end{array}$ \\
\hline $\begin{array}{l}\text { Science/Image } \\
\text { processing }\end{array}$ & Euclid NIR & $\begin{array}{l}\text { Near-Infra-Red image sensor } \\
\text { data processing }\end{array}$ \\
\hline $\begin{array}{l}\text { Optical } \\
\text { Observation }\end{array}$ & GO3S & $\begin{array}{l}\text { High resolution image } \\
\text { processing algorithm }\end{array}$ \\
\hline Compression & $\begin{array}{l}\text { ESA CCSDS 122 } \\
\text { Image data } \\
\text { compression }\end{array}$ & $\begin{array}{l}\text { Generic Image data } \\
\text { compression algorithm }\end{array}$ \\
\hline $\begin{array}{l}\text { Radio } \\
\text { Observation/Signal }\end{array}$ & ADS-B - OCE & $\begin{array}{l}\text { Automatic dependent } \\
\text { surveillance - broadcast } \\
\text { (ADS-B) algorithm- } \\
\text { surveillance technology in } \\
\text { which an aircraft determines } \\
\text { its position via satellite } \\
\text { navigation }\end{array}$ \\
\hline $\begin{array}{l}\text { Vision Based } \\
\text { Navigation }\end{array}$ & $\begin{array}{l}\text { GENEVIS (Solver), } \\
\text { OpenCV }\end{array}$ & $\begin{array}{l}\text { Computer Vision library for } \\
\text { space targets }\end{array}$ \\
\hline $\begin{array}{l}\text { Image Classification, } \\
\text { Tensor Flow }\end{array}$ & $\begin{array}{l}\text { Object detection and } \\
\text { classification }\end{array}$ \\
\hline
\end{tabular}

operation and space exploration, including landing and rover navigation. In the long term, it is anticipated that increasing artificial intelligence will be required on-board most spacecraft.

The emergence of embedded GPUs with high performance and low power consumption presents a real opportunity to drastically increase the on-board processing capability and satisfy the future space applications' performance needs.

A first set of applications fitting in the above-identified areas has been selected and presented in the Table I. Several of these computationally demanding algorithms have already been selected for high performance data processing in current and future missions. Our analysis indicates that all the representative algorithms in the table could be appropriate for GPU acceleration. This is based on the fact that most GPU microarchitectures suffer a significant performance penalty with software exhibiting irregular behaviour, e.g. not accessing consecutive memory positions (memory coalescing) or taking different paths (branch divergence). A first study of those applications shows that they are free of path divergence, while their memory accesses are regular.

Already acquired knowledge and exploitation of those existing and operational software extracts in previous and current parallel studies guarantee both a good starting point for this GPU work and, potentially, an existing baseline for performance and porting effort comparisons. In order to complete the survey and ensure to cover as many potential domains as possible, an internal meeting with all Airbus programs will be organised to identify further algorithms that could be interesting for GPU implementation. 
The space application domain survey includes also the definition of a set of criteria for the selected GPU candidates based on the specific details of particular application domains and missions. For example, Low Earth Orbit (LEO) missions have less stringent availability requirements which allow the use of COTS components, especially if they have reliability features as products that are already qualified for use in industrial or safety critical domains such as the automotive.

Some of the metrics we have identified so far coming from the mission profile include: the availability, usable in space, of the technology/equipment, the algorithms implementation feasibility, the power and thermal constraint of the mission, the I/O interfaces, the non-recurring development cost for radiation testing and the development of mitigation techniques, the recurring cost of the equipment regarding the number of pieces, and the flexibility to use different algorithms at different steps of the mission timeline and the update of the algorithm for correction or extension.

\section{GPU TAXONOMY}

In this Section, we present a taxonomy of mobile GPUs, summarised in Fig 2 in order to better explain the various options of potential candidates for the space domain.

At the top level we classify GPUs as either embedded or high-performance and focus just on the former category, since the latter was covered in previous studies [1][2].

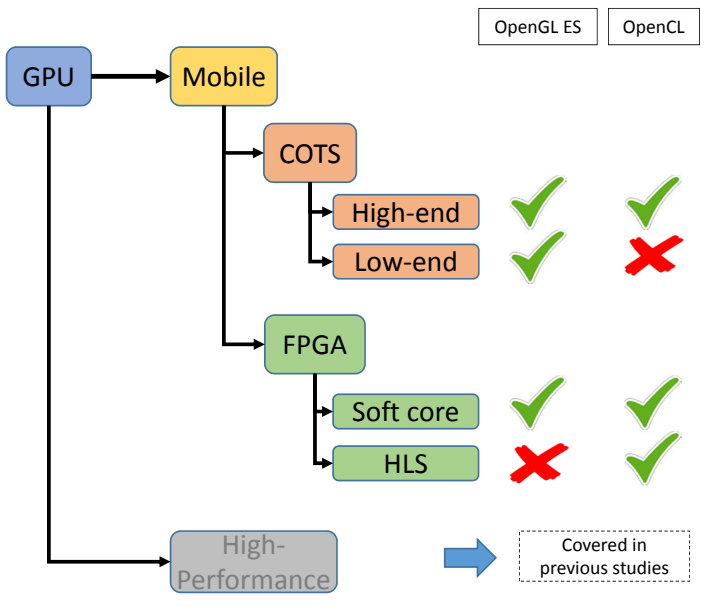

Fig. 2. GPU Taxonomy

The first difference we observed in the mobile GPU market is that, unlike the desktop GPU market which is dominated by 3 major players (NVIDIA, AMD and in lesser degree Intel), the embedded GPU market features several big GPU IP providers. Most of them are European (ARM, Imagination Technologies) or started as European (Adreno was initially designed under the Imageon brand name by the Finnish company BitBoys, now Qualcomm Finland, after acquisition by ATI and AMD). Similarly Broadcom's VideoCore used in the low-cost European educational computer Raspberry Pi has been developed by the UK-based Alphamosaic before it was purchased by Broadcom. In addition, ThinkSilicon which specialises on GPUs for IoT devices is based in Greece.
Another key element in our work is that based on previous works [6][13][14] we were able to extend the survey beyond the obvious choice of OpenCL programmable devices (right part in Fig 2), which allows better covering the available embedded GPU market and providing a wider spectrum for the appropriate selection of embedded GPUs. Note that in order to complete the survey, especially regarding the potential embedded GPU IP acquisition to make a rad-hard chip, it is required to contact the various vendors, which requires a long administrative process that it is currently under its way.

Mobile GPUs can be first divided into two broad categories, COTS GPUs and FPGA implementations. In the former category we find mobile GPUs which are implemented as ASICs (Application-Specific Integrated Circuits), typically as part of a larger SoC (System-On-Chip) which usually contains CPUs and other devices e.g. DSPs, embedded memory etc. In the latter category, FPGA implementations allow reconfiguration of the circuitry in order to facilitate hardware development, especially when a product is not fully defined or additional requirements are introduced at a later time. Both categories are interesting for the space domain.

\section{A. COTS GPUs}

COTS designs provide small recurring costs and higher performance compared to FPGA implementations using the same design technology (often specified in $\mathrm{nm}$ ). COTS components are manufactured in advanced state-of-the-art silicon technologies like $10 \mathrm{~nm}$ FinFET since they target widely used consumer devices in order to benefit from the higher performance and power characteristics of those processes. However, the reliability in those markets regarding transient and permanent faults has not been addressed, since these products are not used in critical systems and they are expected to be replaced voluntarily by the users every 2-3 years with later products with more advanced capabilities. Therefore, additional effort is required in order to shield those components from radiation effects either in hardware or in software.

The COTS GPU category can be further subdivided into Low-End and High-End products.

\section{A.1. Low-end GPUs}

Low-end GPUs support only graphics APIs such as OpenGL ES 2 and have a large share of the mobile market [15][16]. These products feature a simpler architectural design, which is incapable of supporting OpenCL but results in lower power consumption. The most prominent example is ARM's Mali$4 X X$, probably the most licensed embedded GPU to date, which has a $20 \%$ of the smartphone market share [17], without including in this market portion other massively produced consumer devices such as single-board-computers, set-topboxes, tv-sets, automotive systems and FPGAs, such as the Zynq UltraScale+. An advantage of such a widely used older device is the maturity of technology and its associated development tools, which provide evidence from use and documentation of their known problems, if not already fixed in later hardware/driver revisions. 
Other products in this category from different vendors are Qualcomm's Adreno 2xx, Broadcomm's VideoCore IV, Imagination Technologies PowerVR SGX and ThinkSilicon's NemaPico and NemaTiny. Although OpenCL support is not provided on low-end GPUs, and cannot be implemented without low-level access to the GPU design, efficient solutions for general purpose computations on them exist [13][14].

A potential issue with the adoption in space of both low and high-end embedded GPUs, is the non-disclosed architectural design of almost all embedded GPU, which prevents getting the required full control over the execution and observability for space qualification. The vendors provide only high-level information about the implementation and all the development tools are closed source. The only GPU with open specification so far is the VideoCore IV from Broadcom, however the limited available development tools from the open source community work at assembly level. This translates to low productivity and high complexity, while there is no debugging or profiling method available.

\section{A.2. High-end GPUs}

GPUs in the high-end of the embedded spectrum support both graphics and compute APIs, such as OpenCL. Such architectures are ARMs Mali T6xx-T8xx and G7X families, Imaginations latest SGX and Rogue families, Qualcomm's Adreno 3xx and above, ThinkSilicon's NemaSmall and Vivante's latest GC series. Despite those architectures theoretically can support an OpenCL runtime, GPU vendors do not always provide a driver. In fact, OpenCL is usually only available to developers for use with certain development kits, while recently Google dropped its use in Android. As a consequence, GPU vendors are less keen to develop, release or support OpenCL drivers for mobile GPUs, unless there is an explicit interest from large companies such as Samsung or Sony for certain GPU models in their flagship products, or from other domains such as supercomputing. Therefore, the selection of a high-end GPU has to involve a deeper analysis than a simple analysis of vendors' products sheets.

Since most of the embedded GPUs target mainly consumer markets, they don't address explicitly safety requirements. The exceptions in this is case are Imagination's PowerVR 6XT (GX6650) GPU found in ASIL-B certified automotive platforms such as the Renesas R-Car H3 and Imagination's latest GPU Series Furian and NVIDIA's Xavier, which are designed targeting ASIL-D certification, the highest assurance level in automotive systems.

\section{B. FPGA Solutions}

FPGA solutions offer lower performance but the underlying COTS FPGA device can be radiation hardened and qualified for space use such as Xilinx's high-density single-event immune V5QV. Moreover, studies of new silicon technologies have shown better reliability than the current technology used in space $(65 \mathrm{~nm})$, increasing the ambitions for use in space.

The Xilinx report about Failures in Time (FIT) on Xilinx FPGA [18], shows an improvement with technologies that shall be confirmed with the next FPGA generation. Such solutions can provide additional advantages for long interplanetary missions. In particular, the FPGA configurability can reduce the time-to-launch of a several year mission, even in the case that the desired hardware accelerator is not fully developed, debugged or tested. Or, in the case that a new more effective image compression algorithm is invented, a hardware accelerator can be reprogrammed to support it, thus reducing the data size and therefore the time for downlink communications. For the above reasons, both potential solutions have their unique advantages, and therefore they are considered in our survey.

This group can be further divided into two categories:

\section{B.1. Soft GPU cores}

Soft embedded GPU cores are implemented in RTL (Register Transfer Level) using a hardware description language such as Verilog or VHDL. The design is then synthesised on the FPGA, where it can be used transparently to the software either using graphics or compute APIs. Some GPU designer firms explicitly offer evaluation solutions for certain FPGA devices such as Think Silicon with their high-end NEMA GPU and low-end Think2.5D products for Xilinx's Zynq platforms. However, the feedback we have received from all commercial GPU IP vendors so far is that most of the high-end embedded GPUs exceed the capacity of existing FPGAs and only reduced configurations of such designs can fit on very expensive ( $\sim 50 \mathrm{~K}$ euros) FPGA development boards. In addition, according to the vendors the achieved target frequencies of such designs on the FPGA are very low and in conjuction with the reduced configuration, they result in very low performance compared to their ASIC implementation. Therefore, we do not suggest using commercial GPU IP cores for FPGA implementation but only for rad-hard ASICs.

Open source research-oriented soft-GPU cores are also available, such as MIAW [19] and FlexGrip [20], which implement AMD and NVIDIA like GPU microarchitectures or FGPU [21] which does not resemble any commercial GPU. However, these cores do not have a low-power GPU microarchitecture - such as tile-based and deferred rendering architectures [6] -, only implement a subset of the instruction sets (typically limited to integer instructions) and support only compute APIs, not graphics as well. Moreover, the projects come with limited-functionality development tools, and without debugging or profiling capabilities, while most of them are not maintained any more and therefore lack any type of support. Besides these problems, the most important roadblock for using these designs in space is their licensing conditions. Being GPL-licensed for the majority of the cases, using them in a commercial setup with proprietary hardware IP blocks such as SpaceWire, would require the release of the RTL code of the entire platform. For this reason, open source GPU designs are not suitable for this domain.

\section{B.2. High-Level Synthesis}

Modern FPGAs also support OpenCL using High-Level Synthesis (HLS). Such products translate OpenCL to custom 
circuits, which are configured for execution on the FPGA fabric. Although this is not a GPU solution per se, this solution is based on OpenCL, which provides the same software interface as a high-end embedded GPU or soft-GPU core. Such products are both available from Xilinx and Altera, including recent Intel's HARP prototypes, which integrate both a CPU and an FPGA in the same chip. The reconfiguration of this solution provides the advantage that the hardware resources of the FPGA have the potential to be utilised more effectively among various algorithms compared to a fixeddesign soft-GPU solution. However, FPGA reconfiguration (flashing) takes much longer than executing different kernels on a GPU, and although FPGAs are currently used in space missions, this functionality has never been used before.

High-level synthesis in OpenCL facilitates significantly the development and debugging effort compared to a hardware description language. However, the efficiency of the generated circuitry from the high-level synthesis hast to be evaluated. Moreover, existing space qualified FPGAs such as Xilinx's V5QV do not support high-level synthesis. Finally, our analysis indicates that the existing HLS tools are unable to get the same OpenCL code and run it unmodified on an FPGA, either because they require additional glue code between the host and accelerator side or because the kernel code has to be heavily modified and annotated, so that the generated hardware is efficient, in a degree that it will have nothing in common with the original OpenCL code. For this reason, we have concluded that investigating further this path is beyond the scope our project, which is focused only on embedded GPUs, and it is more subject to a future project related to ASIC hardware design for space.

\section{GPU Survey Summary}

Based on the 4 categories of the embedded GPUs which we have identified in our taxonomy, our analysis indicates that the FPGA path should not be pursuited further for implementing commercial COTS GPU designs, nor open source GPU designs. HLS has potential, but it has been deemed out of the scope of this project.

Therefore only commercial embedded GPU devices are considered for the next project phases, either in their COTS SoC implementations or in IP for potential use in future radhard ASICs. Both high-end and low-end products can be used, although they offer different trade-offs in performance, energy efficiency, programming interfaces, maturity, development and debugging tools, open specification and functional safety. Based on the additional vendor information we will collect in the near future and the importance of each of the trafeoffs, we will select the most appropriate embedded GPU candidates for evaluation and comparison with existing on-board devices.

\section{Current and Future Project Work}

Next, we will perform the experimental evaluation of various embedded GPUs from the ones mentioned earlier. The experimental evaluation involves measuring the performance and the power efficiency. We are currently finishing porting algorithms from the representative space application list to the target GPUs and expect to have experimental results by the conference date to include in our presentation. In order to cope with the multiple categories of GPUs and their different programming APIs, we envision the use of Brook Auto [14] which can support multiple back-ends and can facilitate the certification/qualification of the GPU software.

For the comparison, we will establish a set of trade-off criteria, not only based on the performance efficiency, but also on other properties that affect the adoption of GPUs in space, such as the availability and the capabilities of development and profiling tools, the radiation tolerance and the total cost of the hardware and qualification to name a few. All results will be normalised on the current space silicon technology $(65 \mathrm{~nm})$ and will be compared with existing on-board devices.

The comparison will result in the selection of the most appropriate GPU target which will be used to implement a demonstrator with an ESA space application. Moreover, in case that the most appropriate device is COTS, a software reliability plan will be proposed. Finally, the study will conclude with the definition of the roadmap for the adoption of this target in the space domain.

\section{SUMMARY}

In this paper we described the goals and the preliminary results of the GPU4S project, which studies the applicability of embedded GPUs in the space domain.

From our early survey results in space applications and domains, we have identified so far that GPUs are appropriate for a wide range of algorithms from several domains like vision based navigation, image processing, neural network processing and signal processing, and that depending on the particular mission, there are different characteristics like reliability requirement and thermal which need to be taken into account for the selection of the candidate GPUs.

Regarding the embedded GPU domain we have seen that several embedded GPU IP options are potential candidates, most of which European, but each one offers different tradeoffs which have to be further evaluated in the next steps of the project, once all information is obtained, in order to perform the final selection of the platforms that will be evaluated experimentally. However, our analysis indicates that soft GPU IP solutions on FPGA as well as high-level synthesis are not appropriate for further exploration in this project (HLS) or at all (soft GPUs).

\section{ACKNOWLEDGMENTS}

This work has received funding from the the European Space Agency (ESA) under the GPU4S (GPU for Space) Project, answer to the ESA ITT AO/1-9010/17/NL/AF tender with title "Low Power GPU Solutions For High Performance On-Board Data Processing" and from the European Research Council (ERC) under the European Union's Horizon 2020 research and innovation programme (grant agreement No. 772773). This work has also been partially 
supported by the Spanish Ministry of Economy and Competitiveness (MINECO) under grant TIN2015-65316-P and the HiPEAC Network of Excellence. MINECO partially supported Leonidas Kosmidis under Juan de la Cierva Formación postdoctoral fellowship (FJCI-2017-34095) and Jaume Abella under Ramon y Cajal postdoctoral fellowship (RYC-201314717).

\section{REFERENCES}

[1] D. Gonzalez-Arjona and G. Furano, "High-Performance Avionics Solution for Advanced and Complex GNC Systems for ADR (HIPNOS)," TEC-ED \& TEC-SW Final Presentation Days at ESA, December 2017.

[2] G. Lentaris, K. Maragos, I. Stratakos, L. Papadopoulos, O. Papanikolaou, D. Soudris, M. Lourakis, X. Zabulis, D. Gonzalez-Arjona, and G. Furano, "High-Performance Embedded Computing in Space: Evaluation of Platforms for Vision-Based Navigation," Journal of Aerospace Information Systems, vol. 15, no. 4, pp. 178-192, February 2018.

[3] "Bringing Console Quality Lighting to Mobile (Presented by Imagination Technologies), Game Developer Conference 2014," http://www. gdcvault.com/play/1020691/Bringing-Console-Quality-Lighting-to, Last accessed: 24-06-2019.

[4] N. Rajovic, P. M. Carpenter, I. Gelado, N. Puzovic, A. Ramirez, and M. Valero, "Supercomputing with commodity CPUs: Are mobile SoCs ready for HPC?" in SC '13: Proceedings of the International Conference on High Performance Computing, Networking, Storage and Analysis, Nov 2013, pp. 1-12.

[5] J. A. Ross, D. A. Richie, S. J. Park, D. R. Shires, and L. L. Pollock, "A case study of OpenCL on an Android mobile GPU," in 2014 IEEE High Performance Extreme Computing Conference (HPEC), Sep. 2014, pp. 1-6.

[6] M. M. Trompouki and L. Kosmidis, "Optimisation Opportunities and Evaluation for GPGPU applications on Low-End Mobile GPUs," in Design, Automation Test in Europe Conference Exhibition (DATE), 2017.

[7] A. Behcet, "Alpha Magnetic Spectrometer (AMS02) experiment on the International Space Station (ISS)," Nuclear Science and Techniques, vol. 14, pp. 182-194, August 2003.

[8] NASA, “Alpha Magnetic Spectrometer - 02," https://ams.nasa.gov, Last accessed: 24-06-2019.
[9] S. Kawamoto, Y. Ohkawa, H. Okamoto, K. Iki, T. Okumura, Y. Katayama, M. Hayashi, Y. Horikawa, H. Kato, N. Murakami, T. Yamamoto, K. Inoue, and M. Ohnishi, "Current Status of Research and Development on Active Debris Removal at JAXA," 7th European Conference on Space Debris (SDC7), 2017.

[10] O. Notebaert, J. Franklin, V. Lefftz, J. Moreno, M. Patte, M. Syed, and A. Wagner, "Way Forward for High Performance Payload Processing Development," in Data Systems in Aerospace (DASIA), 2012.

[11] M. Patte and O. Notebaert, "Enabling Technologies for Efficient High Performance Processing in Space Applications," in European Conference for Aeronautics and Space Sciences (EUCASS), 2015.

[12] B. Glass and R. Jansen, Eds., International Workshop on Analogue and Mixed-Signal Integrated Circuits for Space Applications (AMICSA) 2016, Gothenburg, Sweden. ESA, 2016.

[13] M. M. Trompouki and L. Kosmidis, "Towards General Purpose Computations on Low-end Mobile GPUs," in Design, Automation Test in Europe Conference Exhibition (DATE), 2016.

[14] M. M. Trompouki and L. Kosmidis, "Brook Auto: High-Level Certification-Friendly Programming for GPU-powered Automotive Systems," in Proceedings of the 55th Annual Design Automation Conference (DAC), 2018.

[15] Khronos, "OpenGL ES Overview," 2018, http://www.khronos.org/ opengles, Last accessed: 24-06-2019.

[16] Google Developers, "OpenGL ES Version," 2018, https://developer android.com/about/dashboards/index.html, Last accessed: 24-06-2019.

[17] ARM, "Mali-400," 2018, https://developer.arm.com/products/ graphics-and-multimedia/mali-gpus/mali-400-gpu, Last accessed: 24-06-2019.

[18] Xilinx, "Device Reliability Report- Xilinx UG116 (v10.5.2)."

[19] R. Balasubramanian, V. Gangadhar, Z. Guo, C.-H. Ho, C. Joseph, J. Menon, M. P. Drumond, R. Paul, S. Prasad, P. Valathol, and K. Sankaralingam, "Enabling GPGPU Low-Level Hardware Explorations with MIAOW: An Open-Source RTL Implementation of a GPGPU," ACM Trans. Archit. Code Optim., vol. 12, no. 2, Jun. 2015.

[20] K. Andryc, M. Merchant, and R. Tessier, "FlexGrip: A soft GPGPU for FPGAs," in International Conference on Field-Programmable Technology $(F P T), 2013$.

[21] M. Al Kadi, B. Janssen, and M. Huebner, "FGPU: An SIMTArchitecture for FPGAs," in Proceedings of the 2016 ACM/SIGDA International Symposium on Field-Programmable Gate Arrays (FPGA), 2016. 